\title{
Free Vibration of Axially Moving Functionally Graded Nanoplates Based on the Nonlocal Strain Gradient Theory
}

\author{
Cheng Li \\ Department of Vehicle Engineering, School of Rail Transportation, Soochow University, Suzhou, Jiangsu 215131, \\ China. \\ MOE Key Lab of Disaster Forecast and Control in Engineering, Jinan University, Guangzhou 510632, Guangdong, \\ China. \\ P. Y. Wang, Q. Y. Luo and S. Li ${ }^{\dagger}$ \\ Department of Vehicle Engineering, School of Rail Transportation, Soochow University, Suzhou, Jiangsu 215131, \\ China. \\ ${ }^{\dagger}$ Corresponding author
}

(Received 17 July 2020; accepted 5 October 2020)

Using the nonlocal strain gradient theory, we explore vibration behaviours of initially bidirectional tensioned functionally graded nanoplates with axial speed. The governing equation of motion can be obtained based on the differential type of nonlocal strain gradient constitutive relation, which characters the dynamics of nanostructures containing kinematic relation. The simply supported boundary constraints on four sides are considered and subsequently the numerical results are determined. It shows that natural frequencies of axially moving nanoplates decrease when increasing the axial speed and the nonlocal parameter. Hence the nonlocal and kinematic factors cause the natural frequencies to decrease or, weaken the equivalent bending rigidity. On the other hand, natural frequencies increase with an increase in the axial tension and material characteristic scale. Hence the strain gradient and tensile stress factors cause the natural frequencies to increase or, strengthen the equivalent bending rigidity. In addition, the natural frequencies get higher with a larger aspect ratio of the functionally graded nanoplate. The larger one between the nonlocal parameter and the material characteristic scale plays a dominant role in the softening and stiffening mechanisms of the nonlocal strain gradient effect. In case of the same magnitude of the nonlocal parameter and the material characteristic scale, the softening and hardening phenomena disappear. The equivalent bending rigidity neither increases nor decreases in such a situation, and its value degenerates to the classical one.

\section{INTRODUCTION}

The mechanical property of nanomaterials and nanostructures plays an important role in the design and control of nano-devices such as nano-electro-mechanical systems and nanorobots, which have attracted extensive attention from related scientists and engineers. ${ }^{1,2}$ The common nanomaterials include carbon nanotubes, ${ }^{3}$ graphene, ${ }^{4}$ hexagonal boron nitride, ${ }^{5}$ metal nanoparticles and so on. The common nanostructures include nanowires or nanobeams, ${ }^{6}$ nanoplates, ${ }^{7}$ nanospheres ${ }^{8}$ as well as composite structures reinforced by nanomaterials. ${ }^{9}$ Among various different mechanical properties, the dynamic characteristics of nanomaterials and nanostructures occupy a very important position. ${ }^{10}$ As one of the focusing issues, the dynamic response is a typical feature in the operation of nano-devices that needs to be paid attention to. Due to the limit of the size of nanomaterials and nanostructures, the experimental studies on the dynamic characteristics of nanomaterials and nanostructures are relatively complex, and it is difficult to extract and measure the dynamic characteristics accurately. Therefore, theoretical models ${ }^{11}$ and atomic simulations $^{12}$ are the main approaches to study the dynamics of nanomaterials and nanostructures in the literature.
In terms of theoretical models, the nonlocal theory proposed by Eringen ${ }^{13-15}$ is one of the widely used theoretical methods in recent decades. For example, Xu and Deng ${ }^{16}$ presented the nonlocal and surface effects in the adsorption-induced resonance of nanobeams using the nonlocal theory and they also revealed the influence of the adsorption density. To solve the derived integral nonlocal governing equation, Lim et al. ${ }^{17}$ developed a new nonlocal finite element method to investigate the torsional statics and dynamics of circular nanotubes/nanorods subjected to concentrated and distributed torques, respectively. Yang et al. ${ }^{18}$ investigated the nonlinear coupling effects of thermal loading and surface stress on pull-in instability of electrically actuated circular nanoplates based on the nonlocal theory. Jalaei and Arani ${ }^{19}$ examined static properties and dynamic behaviours of orthotropic double-layered graphene sheets resting on visco-Pasternak foundation under a longitudinal magnetic field and mechanical transverse loads. The first order shear deformation theory and Eringen's nonlocal theory were employed and two solution procedures were applied to numerically solve the governing equations of motion for graphene sheets with arbitrary boundary conditions. However, in the literature on nanostructural dynamics based on the nonlocal 
theory, there were two kinds of theoretical models with opposite predictions..$^{20}$ One is called the softening model in which the natural frequency of nanostructures decreases with the enhancement of nonlocal effect, ${ }^{18}$ and the other is the stiffening model in which the natural frequency increases with enhancing the nonlocal effect. ${ }^{21}$ The inconsistency in the predictions of the two models has caused confusion. Shen and $\mathrm{Li}^{22}$ resolved such a puzzled problem and showed the adaptability of the two models by establishing a semi-continuum model of nanostructures. Furthermore, based on the nonlocal theory, Lim et al. ${ }^{23}$ proposed a nonlocal strain gradient theory by considering both the nonlocal effect of strains and first-order strain gradient, and the gradient effect of classical and nonlocal stresses. In case of omitting the strain gradient effect, the nonlocal strain gradient theory returns to Eringen's nonlocal theory. In case of omitting the nonlocal effect, the nonlocal strain gradient theory returns to the general strain gradient theory. Based on the nonlocal strain gradient theory, it is shown that there are two modes including strengthening and weakening in nanostructural dynamics, depending on the strength relationship between the nonlocal parameter and the material characteristic scale. ${ }^{24}$ The two modes correspond to both the nonlocal softening model and the stiffening model, respectively. Using the nonlocal strain gradient theory, Sahmani and Aghdam ${ }^{25}$ and Sahmani et al. ${ }^{26}$ presented the nonlinear instability of functionally graded multilayer graphene platelet-reinforced nanoshells subjected to axial compressive loads, and the size-dependent behaviours in nonlinear large-amplitude vibration of functionally graded porous nanoplates reinforced with graphene platelets, respectively.

The nonlocal strain gradient theory comprehensively considers the nonlocal effect and strain gradient effect of nanomaterials and nanostructures, so it has received great attention in recent years. There have been a large number of published papers dealing with the application of this theory to the dynamics of nanostructures. ${ }^{27-35}$ For example, Chen et al. ${ }^{29}$ studied the stability mechanism and nonlinear dynamics of a nanobeam with a time-dependent axial load based on the nonlocal strain gradient theory. Mirjavadi et al. ${ }^{34}$ investigated the transient responses of a porosity-dependent functionally graded nanobeam subjected to different impulsive loadings. Different porosity distribution types were considered and the inverse Laplace transform approach was adopted to determine the dynamic deflections. Mir and Tahani ${ }^{35}$ presented the nonlinear dynamics and chaotic behaviours of graphene-based resonators. A nanoplate model with von Karman-type geometric nonlinearity was established and the Galerkin decomposition method and Melnikov integral method were utilized to derive analytical criteria and chaotic regions of the system. As we know, the classical local theory that deals with macro-scale structures does not take into account any microscopic effects, and the nonlocal theory only considers the nonlocal stress corresponding to the strain itself but ignores the nonlocal effect of the strain gradient, while the strain gradient theory only considers the strain gradient effect of the local stress but ignores the influence of the nonlocal stress. Therefore, the classical local theory, the nonlocal theory and the strain gradient theory have certain limitations in showing the special attributes of nanostructures. In fact, the classical local constitutive rela- tion does not include any information about the internal characteristic scales of the material, while the nonlocal theory and the strain gradient theory respectively contain one characteristic scale parameter. The nonlocal strain gradient theory, by contrast, is more comprehensive in modeling and predicting, where the nonlocal effect of strain and its gradient, and the strain gradient effect of local and nonlocal stresses are arrived at by introducing two characteristic scale parameters into the constitutive equations. A large amount of literature has confirmed that the nonlocal strain gradient theory is more suitable for explaining the microscopic mechanical phenomena of nanostructures and further revealing the physical mechanisms of mechanical properties at nanoscale. More accurate theoretical models and results for nanostructures can be obtained based on the nonlocal strain gradient theory. ${ }^{24-26}$ Consequently, the nonlocal strain gradient theory is applied directly to nanostructural dynamics in this paper.

In addition to the dynamic characteristics of nanostructures, their own kinematic factors cannot be negligible. This is because a considerable number of nanostructures, such as nanorobots, are in motion during the work process. In the fields of medicine, food, microelectronics and so on, nanorobots generally keep linear or reciprocating motion, and sometimes the key parts in the nanorobots are accompanied by rotation. ${ }^{36}$ Therefore, it is necessary to demonstrate the dynamics of moving nanostructures. At present, the dynamic responses of onedimensional and two-dimensional nanostructures with axial motion have been reported. ${ }^{36-39}$ However, most of them focus on the nanostructures composed of homogeneous materials. In fact, with the increasing requirements of extreme engineering applications, such as high temperature, high pressure, high strain rate and other external factors, homogeneous nanostructures have been difficult to meet the demanding functional requirements. In this case, functional gradient structures and even functional gradient nanostructures emerge, and have been successfully designed and prepared. ${ }^{40-43}$ The functional gradient nanostructure has the dual characteristics of functional gradient structures and nanomaterials, and it is one of the research hotspots in recent years. ${ }^{44,45}$ Considering that the thickness of components in nanorobots or other equipment is very thin, say generally in the order of less than ten nanometers, which is far less than the length and width, but the width is not significantly less than the length, so it is more general to simplify the key components of nanorobots into plate-like nanostructures rather than nanobeams. Moreover, because of the extremely small thickness, the dynamic characteristics change little along the thickness direction, so the influence of the thickness on the dynamics can be neglected. For the first time, Shen et al..$^{46}$ introduced nonlocal strain gradient theory into the axially moving functionally graded nanoplates to study the dynamic stability, but lacked the systematic analysis on free vibration. Considering that the axially moving structure is often subjected to initial axial tensions in practical engineering, ${ }^{47}$ this paper studies the transverse free vibration of axially moving functionally graded nanoplates subjected to the initial tensile force.

It is worth mentioning that, although there have been some studies on the dynamics and stability of axially moving nanoplates, ${ }^{48}$ these were only based on the nonlocal theory, without the consideration of the functional gradient material 
and the more comprehensive nonlocal strain gradient effect. On the other hand, there have also been some studies on the dynamics and stability of functionally graded nanoplates 49,50 based on the nonlocal strain gradient theory. Nevertheless, they did not consider the kinematic factor of the nanoplate itself that is indispensable in modeling the components made of functionally graded materials in nanorobots. The main innovation of this paper is to study the vibration characteristics of axially moving functionally graded nanoplates with biaxial tensions using the nonlocal strain gradient theory. Effects of various parameters on the dynamic characteristics of axially moving functionally graded nanoplates are presented, including the intrinsic parameters such as nonlocal parameter, material characteristic scale, and the external parameters such as aspect ratio, axial speed, initial biaxial tension and so on. The research results may provide a theoretical basis for the dynamic and kinematic coupling control of nanorobots containing components made from two-dimensional functional gradient nanomaterials.

\section{THEORETICAL MODEL AND GOVERNING EQUATION OF MOTION}

Based on the concept of the nonlocal strain gradient theory, the basic integral constitutive equations of two-dimensional nanostructures were expressed as ${ }^{23}$

$$
\begin{aligned}
\sigma & =\int_{V} \alpha_{0}\left(x^{\prime}, x, e_{0} a\right) C: \varepsilon^{\prime} d V^{\prime} ; \\
\sigma^{(1)} & =l^{2} \int_{V} \alpha_{1}\left(x^{\prime}, x, e_{1} a\right) C: \nabla \varepsilon^{\prime} d V^{\prime} ; \\
t & =\sigma-\nabla \sigma^{(1)} ;
\end{aligned}
$$

where $\sigma$ and $\sigma^{(1)}$ represented the classical and the nonclassical higher-order nonlocal stresses respectively, $\varepsilon^{\prime}$ was the strain and $t$ was the nonlocal strain gradient total stress, $C$ was the elastic tensor, $\alpha_{0}$ and $\alpha_{1}$ were nonlocal key functions related to strain and first-order strain gradient, respectively. Meanwhile, $e_{0}$ and $e_{1}$ were the traditional and higher-order nonlocal material constants, $a$ was an internal characteristic length, and $l$ was the material characteristic scale introduced to indicate the effect of higher-order strain gradient fields.

Considering the spatial integration of strains and strain gradients shown in Eq. (1), we had difficulty in solving the constitutive equations. Using Laplace operator $\nabla^{2}$, the simplified differential constitutive relation of the nonlocal strain gradient theory was given by ${ }^{23}$

$$
\left[1-(e a)^{2} \nabla^{2}\right] t=C\left(1-l^{2} \nabla^{2}\right) \varepsilon^{\prime} ;
$$

where $e_{0}=e_{1}=e$ was assumed.

Based on the nonlocal strain gradient theory, the governing equation of motion for an axially moving functionally graded nanoplate had already been obtained by Shen et al. ${ }^{46}$ as

$$
\begin{gathered}
\left(1-l^{2} \nabla^{2}\right) D\left(\frac{\partial^{4} w}{\partial x^{4}}+\frac{\partial^{4} w}{\partial y^{4}}+\frac{\partial^{4} w}{\partial x^{2} \partial y^{2}}\right)= \\
{\left[1-(e a)^{2} \nabla^{2}\right]\left[N_{x} \frac{\partial^{2} w}{\partial x^{2}}+N_{y} \frac{\partial^{2} w}{\partial y^{2}}-\right.} \\
\left.\rho(z) h\left(v^{2} \frac{\partial^{2} w}{\partial x^{2}}+2 v \frac{\partial^{2} w}{\partial x \partial t}+\frac{\partial^{2} w}{\partial t^{2}}\right)\right]
\end{gathered}
$$

where $w$ was the out-of-plane displacement, $D$ was the equivalent bending rigidity, $v$ was axial speed, $\rho$ was mass density, $h$ was thickness of the functionally graded nanoplate, and $N_{x}$ and $N_{y}$ were initial biaxial tensions along $x$ and $y$ axes, respectively. Note that the biaxial tensions may be produced by the change of temperature or the nanostructural assembling prestress in nanorobots or other mobile nano-devices. In fact, it is difficult to achieve a stable free vibration of axially moving structures without axial tensile forces. Introducing the following dimensionless parameters

$$
\begin{gathered}
\bar{x}=\frac{x}{L_{a}} ; \quad \bar{y}=\frac{y}{L_{b}} ; \quad \bar{w}=\frac{w}{h} ; \quad \lambda=\frac{L_{a}}{L_{b}} ; \\
P_{x}=\frac{N_{x} L_{a}^{2}}{D} ; \quad P_{y}=\frac{N_{y} L_{a}^{2}}{D} ; \quad \tau=\frac{e a}{L_{a}} ; \quad \zeta=\frac{l}{L_{a}} ; \\
\xi=\sqrt{\frac{\rho(z) h}{D}} L_{a} v ; \quad \bar{t}=\sqrt{\frac{D}{\rho(z) h L_{a}^{4}}} t
\end{gathered}
$$

where $\bar{x}, \bar{y}$ and $\bar{w}$ were non-dimensional axial coordinates and out-of-plane displacement, $\lambda$ was the aspect ratio, $P_{x}$ and $P_{y}$ were non-dimensional biaxial tensions, $\tau$ and $\zeta$ were nondimensional nonlocal and material characteristic scale parameters, which denoted the normalized nonlocal and strain gradient effects in the nonlocal strain gradient theory, respectively, $\xi$ was the non-dimensional axial speed, and $\bar{t}$ was nondimensional time. As a result, the dimensionless form of Eq. (3) was rewritten as

$$
\begin{gathered}
\zeta^{2} \frac{\partial^{6} \bar{w}}{\partial \bar{x}^{6}}+3 \zeta^{2} \lambda^{2} \frac{\partial^{6} \bar{w}}{\partial \bar{x}^{4} \partial \bar{y}^{2}}+3 \zeta^{2} \lambda^{4} \frac{\partial^{6} \bar{w}}{\partial \bar{x}^{2} \partial \bar{y}^{4}}+\zeta^{2} \lambda^{6} \frac{\partial^{6} \bar{w}}{\partial \bar{y}^{6}}- \\
{\left[1+\tau^{2}\left(P_{x}-\xi^{2}\right)\right] \frac{\partial^{4} \bar{w}}{\partial \bar{x}^{4}}-} \\
{\left[2 \lambda^{2}+\tau^{2} \lambda^{2}\left(P_{x}+P_{y}-\xi^{2}\right)\right] \frac{\partial^{4} \bar{w}}{\partial \bar{x}^{2} \partial \bar{y}^{2}}-} \\
\lambda^{4}\left(1+\tau^{2} P_{y}\right) \frac{\partial^{4} \bar{w}}{\partial \bar{y}^{4}}+2 \tau^{2} \xi \frac{\partial^{4} \bar{w}}{\partial \bar{x}^{3} \partial \bar{t}}+\tau^{2} \frac{\partial^{4} \bar{w}}{\partial \bar{x}^{2} \partial \bar{t}^{2}}+ \\
2 \tau^{2} \xi \lambda^{2} \frac{\partial^{4} \bar{w}}{\partial \bar{x} \partial \bar{y}^{2} \partial \bar{t}}+\tau^{2} \lambda^{2} \frac{\partial^{4} \bar{w}}{\partial \bar{y}^{2} \partial \bar{t}^{2}}+\left(P_{x}-\xi^{2}\right) \frac{\partial^{2} \bar{w}}{\partial \bar{x}^{2}}+ \\
P_{y} \lambda^{2} \frac{\partial^{2} \bar{w}}{\partial \bar{y}^{2}}-2 \xi \frac{\partial^{2} \bar{w}}{\partial \bar{x} \partial \bar{t}}-\frac{\partial^{2} \bar{w}}{\partial \bar{t}^{2}}=0
\end{gathered}
$$

It was noticed that the common theoretical model based on the nonlocal strain gradient elasticity and Kirchhoff plate theory can be recovered in case of vanishing the additional considerations including the axial speed and biaxial tensions, shown as

$$
\begin{gathered}
\zeta^{2} \frac{\partial^{6} \bar{w}}{\partial \bar{x}^{6}}+3 \zeta^{2} \lambda^{2} \frac{\partial^{6} \bar{w}}{\partial \bar{x}^{4} \partial \bar{y}^{2}}+3 \zeta^{2} \lambda^{4} \frac{\partial^{6} \bar{w}}{\partial \bar{x}^{2} \partial \bar{y}^{4}}+\zeta^{2} \lambda^{6} \frac{\partial^{6} \bar{w}}{\partial \bar{y}^{6}}- \\
\frac{\partial^{4} \bar{w}}{\partial \bar{x}^{4}}-2 \lambda^{2} \frac{\partial^{4} \bar{w}}{\partial \bar{x}^{2} \partial \bar{y}^{2}}-\lambda^{4} \frac{\partial^{4} \bar{w}}{\partial \bar{y}^{4}}+\tau^{2} \frac{\partial^{4} \bar{w}}{\partial \bar{x}^{2} \partial \bar{t}^{2}}+ \\
\tau^{2} \lambda^{2} \frac{\partial^{4} \bar{w}}{\partial \bar{y}^{2} \partial \bar{t}^{2}}-\frac{\partial^{2} \bar{w}}{\partial \bar{t}^{2}}=0
\end{gathered}
$$

One can further obtain the classical non-dimensional vibration equation for square plates by ignoring the internal characteristic scale parameters

$$
\frac{\partial^{4} \bar{w}}{\partial \bar{x}^{4}}+2 \frac{\partial^{4} \bar{w}}{\partial \bar{x}^{2} \partial \bar{y}^{2}}+\frac{\partial^{4} \bar{w}}{\partial \bar{y}^{4}}+\frac{\partial^{2} \bar{w}}{\partial \bar{t}^{2}}=\nabla^{4} \bar{w}+\frac{\partial^{2} \bar{w}}{\partial \bar{t}^{2}}=0 .
$$


For a nanoplate, the time-dependent out-of-plane displacement was written by

$$
\bar{w}(\bar{x}, \bar{y}, \bar{t})=\bar{W}(\bar{x}, \bar{y}) e^{i \omega_{m n} \bar{t}}
$$

where $\omega_{m n}$ was the complex natural frequency of axially moving functionally graded nanoplates.

The substitution of Eq. (6) into Eq. (5a) can deduce the following equation in terms of natural frequencies and vibration modes

$$
\begin{gathered}
\zeta^{2} \frac{\partial^{6} \bar{W}}{\partial \bar{x}^{6}}+3 \zeta^{2} \lambda^{2} \frac{\partial^{6} \bar{W}}{\partial \bar{x}^{4} \partial \bar{y}^{2}}+3 \zeta^{2} \lambda^{4} \frac{\partial^{6} \bar{W}}{\partial \bar{x}^{2} \partial \bar{y}^{4}}+\zeta^{2} \lambda^{6} \frac{\partial^{6} \bar{W}}{\partial \bar{y}^{6}}- \\
{\left[1+\tau^{2}\left(P_{x}-\xi^{2}\right)\right] \frac{\partial^{4} \bar{W}}{\partial \bar{x}^{4}}-} \\
{\left[2 \lambda^{2}+\tau^{2} \lambda^{2}\left(P_{x}+P_{y}-\xi^{2}\right)\right] \frac{\partial^{4} \bar{W}}{\partial \bar{x}^{2} \partial \bar{y}^{2}}-} \\
\lambda^{4}\left(1+\tau^{2} P_{y}\right) \frac{\partial^{4} \bar{W}}{\partial \bar{y}^{4}}+2 i \tau^{2} \xi \omega_{m n} \frac{\partial^{3} \bar{W}}{\partial \bar{x}^{3}}+ \\
2 i \tau^{2} \xi \lambda^{2} \omega_{m n} \frac{\partial^{3} \bar{W}}{\partial \bar{x} \partial \bar{y}^{2}}+\left(P_{x}-\xi^{2}-\tau^{2} \omega_{m n}^{2}\right) \frac{\partial^{2} \bar{W}}{\partial \bar{x}^{2}}+ \\
\lambda^{2}\left(P_{y}-\tau^{2} \omega_{m n}^{2}\right) \frac{\partial^{2} \bar{W}}{\partial \bar{y}^{2}}-2 i \xi \omega_{m n} \frac{\partial \bar{W}}{\partial \bar{x}}+\omega_{m n}^{2} \bar{W}=0 .
\end{gathered}
$$

\section{NUMERICAL SOLUTIONS AND DISCUSSION}

Taking the axially moving functionally graded nanoplate with simply supported boundary constraints on four sides as an example, we calculate the natural frequencies herein numerically. For simply supported boundary on four sides, the deflection and bending moment at each side should be zero. Therefore, the dimensionless form of boundary conditions is written as

$$
\left\{\begin{array}{l}
\left.\bar{W}(\bar{x}, \bar{y})\right|_{\bar{x}=0,1}=\left.\frac{\partial^{2} \bar{W}(\bar{x}, \bar{y})}{\partial \bar{x}^{2}}\right|_{\bar{x}=0,1}=0 \\
\left.\bar{W}(\bar{x}, \bar{y})\right|_{\bar{y}=0,1}=\left.\frac{\partial^{2} \bar{W}(\bar{x}, \bar{y})}{\partial \bar{y}^{2}}\right|_{\bar{y}=0,1}=0 .
\end{array}\right.
$$

In addition to the classical boundary conditions shown in Eq. (8), the nonlocal boundary condition can be obtained in the variational procedure, given by

$$
\frac{\partial \bar{W}(\bar{x}, \bar{y})}{\partial \bar{t}}+\left.\xi \frac{\partial \bar{W}(\bar{x}, \bar{y})}{\partial \bar{x}}\right|_{\bar{x}=0,1}=\left.\frac{\partial \bar{W}(\bar{x}, \bar{y})}{\partial \bar{x}}\right|_{\bar{x}=0,1}=0
$$

The dimensionless displacement can be separated into two functions with respect to $x$ and $y$, given by

$$
\begin{aligned}
\bar{W}(\bar{x}, \bar{y}) & =\sum_{m=1}^{M} \sum_{n=1}^{N} A_{m n} \psi_{m n}(\bar{x}, \bar{y}) \\
& =\sum_{m=1}^{M} \sum_{n=1}^{N} A_{m n} \phi_{m}(\bar{x}) \varphi_{n}(\bar{y})
\end{aligned}
$$

where $\phi_{m}(\bar{x})$ and $\varphi_{n}(\bar{y})$ are the non-dimensional mode functions of $x$ and $y$ direction, respectively, and $\psi_{m n}(\bar{x}, \bar{y})$ is product of two mode functions.
For a simply supported nanoplate, its $y$-direction mode function can be written by

$$
\varphi_{n}(\bar{y})=\sin n \pi \bar{y} .
$$

So the mode function is expressed as

$$
\psi_{m n}(\bar{x}, \bar{y})=\phi_{m}(\bar{x}) \sin n \pi \bar{y}
$$

Substituting Eq. (12) into Eq. (10), and further substituting the obtained results into Eq. (7) yield

$$
\begin{gathered}
\zeta^{2} \frac{\partial^{6} \phi_{m}(\bar{x})}{\partial \bar{x}^{6}}-\left[3(n \pi)^{2} \zeta^{2} \lambda^{2}+\tau^{2}\left(P_{x}-\xi^{2}\right)+1\right] \frac{\partial^{4} \phi_{m}(\bar{x})}{\partial \bar{x}^{4}}+ \\
2 i \tau^{2} \xi \omega_{m n} \frac{\partial^{3} \phi_{m}(\bar{x})}{\partial \bar{x}^{3}}+\left\{(n \pi)^{2} \lambda^{2}\left[2+\tau^{2}\left(P_{x}+P_{y}-\xi^{2}\right)\right]+\right. \\
\left.3(n \pi)^{4} \zeta^{2} \lambda^{4}+\left(P_{x}-\xi^{2}-\tau^{2} \omega_{m n}^{2}\right)\right\} \frac{\partial^{2} \phi_{m}(\bar{x})}{\partial \bar{x}^{2}}- \\
2 i \xi \omega_{m n}\left[(n \pi)^{2} \tau^{2} \lambda^{2}+1\right] \frac{\partial \phi_{m}(\bar{x})}{\partial \bar{x}}- \\
{\left[(n \pi)^{6} \zeta^{2} \lambda^{6}+(n \pi)^{4} \lambda^{4}\left(1+\tau^{2} P_{y}\right)+\right.} \\
\left.(n \pi)^{2} \lambda^{2}\left(P_{y}-\tau^{2} \omega_{m n}^{2}\right)-\omega_{m n}^{2}\right] \phi_{m}(\bar{x})=0 .
\end{gathered}
$$

The solution of Eq. (13) can be assumed as

$$
\phi_{m}(\bar{x})=C e^{i \beta \bar{x}} .
$$

Substituting Eq. (14) into Eq. (13) yields an algebraic equation, shown as

$$
\begin{aligned}
\zeta^{2} \beta^{6}+ & {\left[3(n \pi)^{2} \zeta^{2} \lambda^{2}+\tau^{2}\left(P_{x}-\xi^{2}\right)+1\right] \beta^{4}-} \\
& 2 i \tau^{2} \xi \omega_{m n} \beta^{3}+\left\{(n \pi)^{2} \lambda^{2}\left[2+\tau^{2}\left(P_{x}+P_{y}-\xi^{2}\right)\right]+\right. \\
& \left.3(n \pi)^{4} \zeta^{2} \lambda^{4}+\left(P_{x}-\xi^{2}-\tau^{2} \omega_{m n}^{2}\right)\right\} \beta^{2}- \\
& 2 i \xi \omega_{m n}\left[(n \pi)^{2} \tau^{2} \lambda^{2}+1\right] \beta+ \\
& {\left[(n \pi)^{6} \zeta^{2} \lambda^{6}+(n \pi)^{4} \lambda^{4}\left(1+\tau^{2} P_{y}\right)+\right.} \\
& \left.(n \pi)^{2} \lambda^{2}\left(P_{y}-\tau^{2} \omega_{m n}^{2}\right)-\omega_{m n}^{2}\right]=0
\end{aligned}
$$

Since Eq. (15) is a sixth-order ordinary differential equation, its solution can be expressed as

$$
\begin{aligned}
\phi_{m}(\bar{x})= & C_{1 m}\left(e^{i \beta_{1 m} \bar{x}}+C_{2 m} e^{i \beta_{2 m} \bar{x}}+C_{3 m} e^{i \beta_{3 m} \bar{x}}+\right. \\
& \left.C_{4 m} e^{i \beta_{4 m} \bar{x}}+C_{5 m} e^{i \beta_{5 m} \bar{x}}+C_{6 m} e^{i \beta_{6 m} \bar{x}}\right)
\end{aligned}
$$

where $C_{j m}(j=1,2,3, \ldots, 6)$ are six unknown coefficients, and $\beta_{j m}(j=1,2,3, \ldots, 6)$ are right six roots of Eq. (15). On the other hand, the boundary conditions can be simplified as

$$
\begin{aligned}
\phi_{m}(0)=\phi_{m}(1) & =\frac{\partial^{2} \phi_{m}(0)}{\partial \bar{x}^{2}}=\frac{\partial^{2} \phi_{m}(1)}{\partial \bar{x}^{2}}=\frac{\partial \phi_{m}(0)}{\partial \bar{x}} \\
& =\frac{\partial \phi_{m}(1)}{\partial \bar{x}}=0 .
\end{aligned}
$$

Therefore, a set of algebraic equations can be derived by substituting Eq. (16) into the boundary conditions Eq. (17). In order to obtain nontrivial solutions of the coefficients or nontrivial solutions of mode functions in Eq. (16), one should set 


\begin{tabular}{c|cccccc}
\hline & 1 & 1 & 1 & 1 & 1 & 1 \\
$e^{i \beta_{1 m}}$ & $e^{i \beta_{2 m}}$ & $e^{i \beta_{3 m}}$ & $e^{i \beta_{4 m}}$ & $e^{i \beta_{5 m}}$ & $e^{i \beta_{6 m}}$ \\
$i \beta_{1 m}$ & $i \beta_{2 m}$ & $i \beta_{3 m}$ & $i \beta_{4 m}$ & $i \beta_{5 m}$ & $i \beta_{6 m}$ \\
$i \beta_{1 m} e^{i \beta_{1 m}}$ & $i \beta_{2 m} e^{i \beta_{2 m}}$ & $i \beta_{3 m} e^{i \beta_{3 m}}$ & $i \beta_{4 m} e^{i \beta_{4 m}}$ & $i \beta_{5 m} e^{i \beta_{5 m}}$ & $i \beta_{6 m} e^{i \beta_{6 m}}$ \\
$\beta_{1 m}^{2}$ & $\beta_{2 m}^{2}$ & $\beta_{3 m}^{2}$ & $\beta_{4 m}^{2}$ & $\beta_{5 m}^{2}$ & $\beta_{6 m}^{2}$ \\
$\beta_{1 m}^{2} e^{i \beta_{1 m}}$ & $\beta_{2 m}^{2} e^{i \beta_{2 m}}$ & $\beta_{3 m}^{2} e^{i \beta_{3 m}}$ & $\beta_{4 m}^{2} e^{i \beta_{4 m}}$ & $\beta_{5 m}^{2} e^{i \beta_{5 m}}$ & $\beta_{6 m}^{2} e^{i \beta_{6 m}}$
\end{tabular} \mid

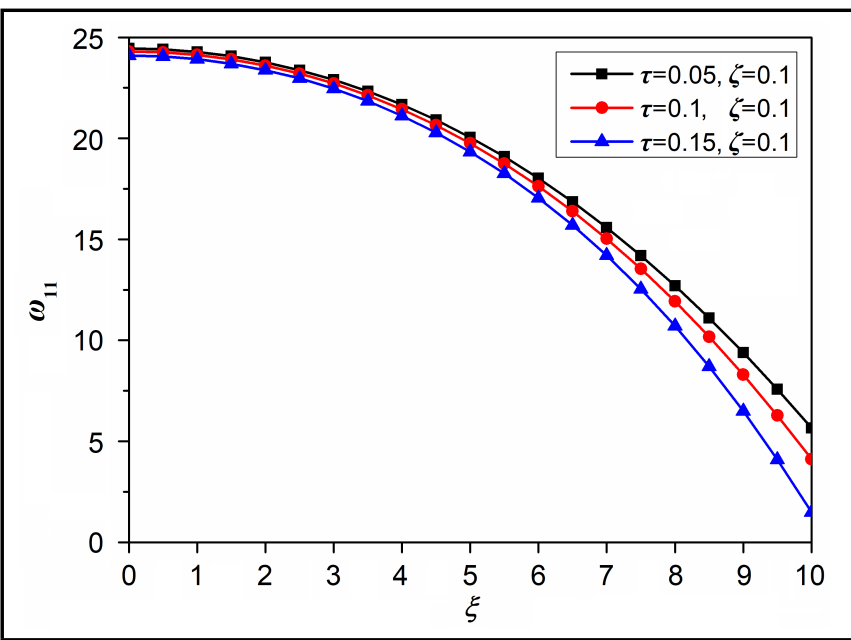

Figure 1. Variation of the first-order frequency of the functionally graded nanoplate with respect to the axial speed $\left(\lambda=1, P_{x}=P_{y}=50\right)$.

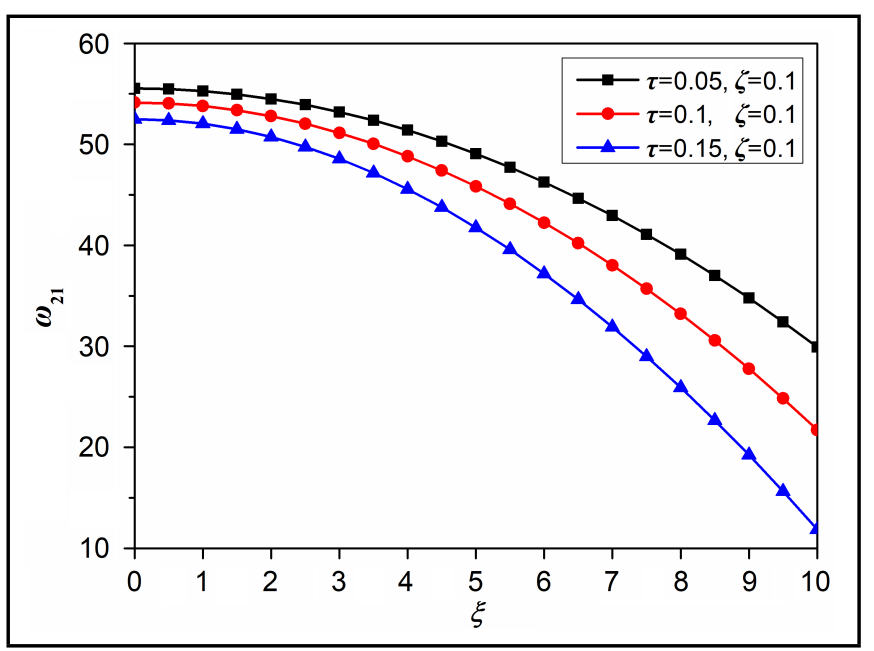

Figure 2. Variation of the second-order frequency of the functionally graded nanoplate with respect to the axial speed $\left(\lambda=1, P_{x}=P_{y}=50\right)$.

the determinant of the coefficient matrix of the algebraic equations to zero, see Eq. (18).

The relation between $\beta_{j m}(j=1,2,3, \ldots, 6)$ is indicated in Eq. (18). Consequently, we can determine the six roots and subsequently get the non-dimensional frequencies by numerically solving the algebraic equations. The variations of nondimensional natural frequencies with respect to various parameters can be demonstrated accordingly.

First, we analyze the influence of the axial speed on the natural frequency, where the frequency mode $n=1, m=1,2$, the dimensionless parameters are $\zeta=0.1, \lambda=1, P_{x}=P_{y}=50$, and the nonlocal parameter $\tau$ are $0.05,0.1,0.15$, respectively. The dimensionless frequency of the functionally graded nanoplates varies with the dimensionless speed $\xi$ as shown in Figs. 1-2. It can be seen that, under a given nonlocal parame-

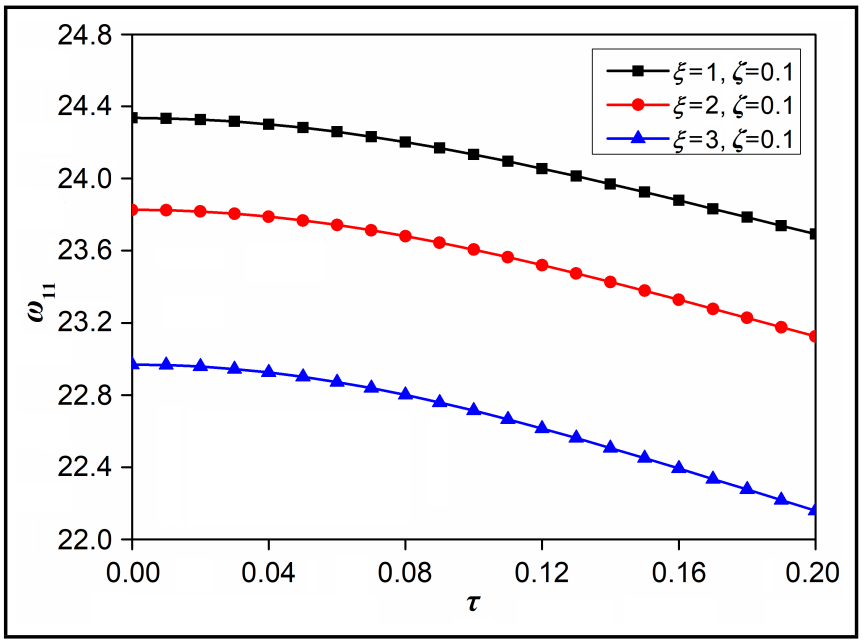

Figure 3. Variation of the first-order frequency of the functionally graded nanoplate with respect to the nonlocal parameter $\left(\lambda=1, P_{x}=P_{y}=50\right)$.

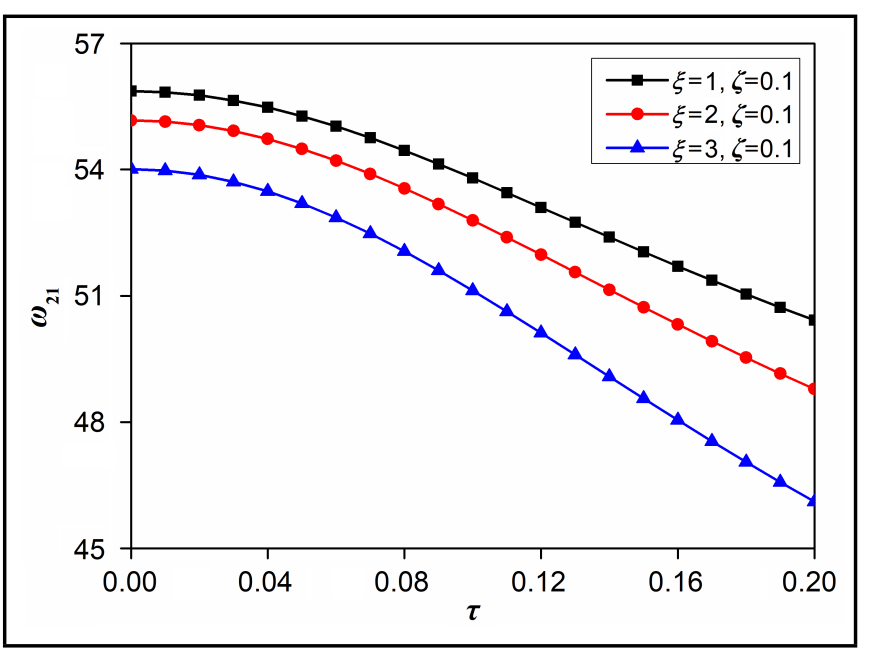

Figure 4. Variation of the second-order frequency of the functionally graded nanoplate with respect to the nonlocal parameter $\left(\lambda=1, P_{x}=P_{y}=50\right)$.

ter, the dimensionless frequency decreases and the decreasing rate gets faster as the dimensionless speed increases. Thus, the stability of the functionally graded nanoplate is reduced as the axial speed increases.

Secondly, in order to explore the effect of the nonlocal parameter $\tau$ on the natural frequency, we assume $\zeta=0.1, \lambda=1$, $P_{x}=P_{y}=50$, and the dimensionless axial speed $\xi=1,2,3$, respectively. Variations of the dimensionless frequency with respect to the nonlocal parameter are displayed in Figs. 3-4. The dimensionless natural frequency decreases with the increase of the nonlocal parameter and the stiffness of the functionally graded nanoplates is continuously decreasing, which reflects the softening behaviour in nonlocal effects. Figure 3 implies the effect of increasing the nonlocal parameter on the reduction rate of natural frequencies, and it can be reflected 


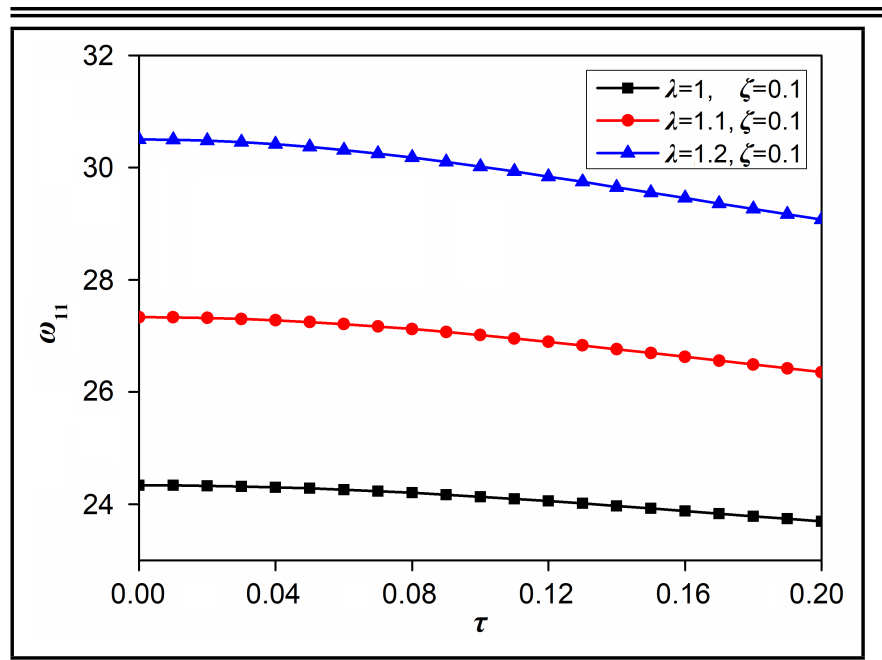

Figure 5. Variation of the first-order frequency of the functionally graded nanoplate with respect to the nonlocal parameter under the different aspect ratio $\left(\xi=1, P_{x}=P_{y}=50\right)$.

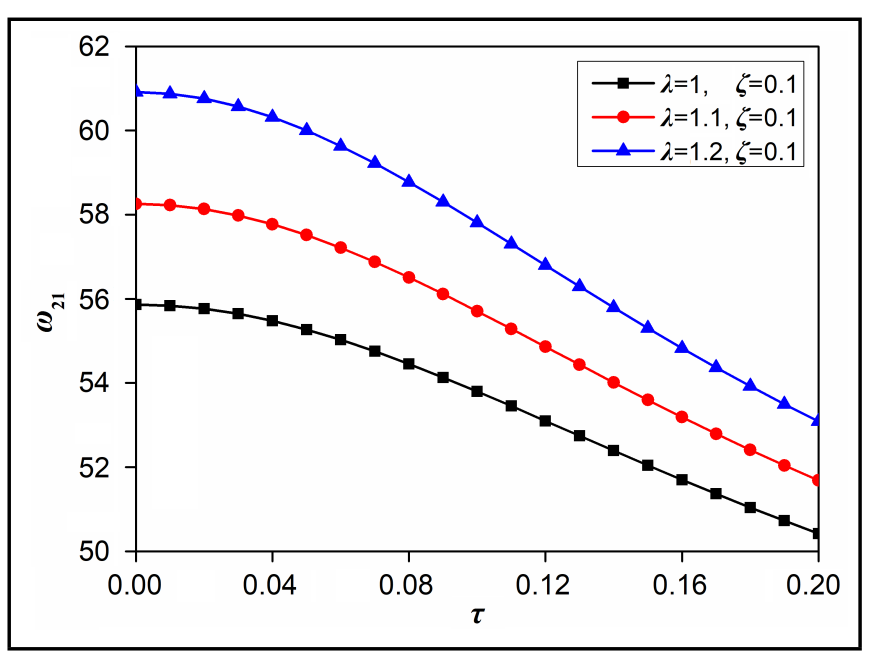

Figure 6. Variation of the second-order frequency of the functionally graded nanoplate with respect to the nonlocal parameter under the different aspect ratio $\left(\xi=1, P_{x}=P_{y}=50\right)$.

prominently in Fig. 4. It can be inferred that the impact of the nonlocal parameter is also more and more significant in highorder frequencies.

Next, in order to analyze the influence of the nonlocal parameter on the natural frequency with different ratios, one can keep the axial speed as a constant, and take the aspect ratio $\lambda=1,1.1,1.2$, respectively. The results are demonstrated in Figs. 5-6. The natural frequency increases with the increase of the aspect ratio, thereby increasing the rigidity of the axially moving functionally graded nanoplate. In Fig. 6, the greater the aspect ratio, the faster the natural frequency decreases with increasing the nonlocal parameter, which has certain guiding significance for the design of rectangular nanoplates.

Figures 7 and 8 indicate variations of natural frequencies of the functionally graded nanoplate with respect to the nonlocal parameter where the material characteristic scale is chosen as $0.05,0.1$, and 0.15 , respectively. It can be seen that when $\zeta$ takes different values, the nonlocal parameter still reflects its stiffness softening effect on the functionally graded nanoplate. When $\zeta$ takes a larger value, the natural frequency appears decreasing faster and faster with the increase of the nonlocal pa-

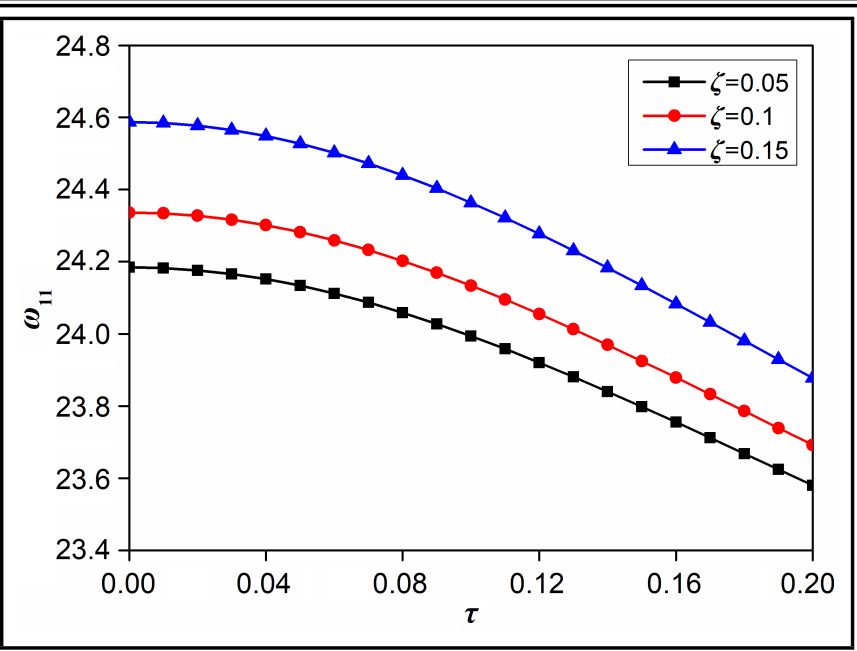

Figure 7. Variation of the first-order frequency of the functionally graded nanoplate with respect to the nonlocal parameter under different material characteristic scales $\left(\xi=1, \lambda=1, P_{x}=P_{y}=50\right)$.

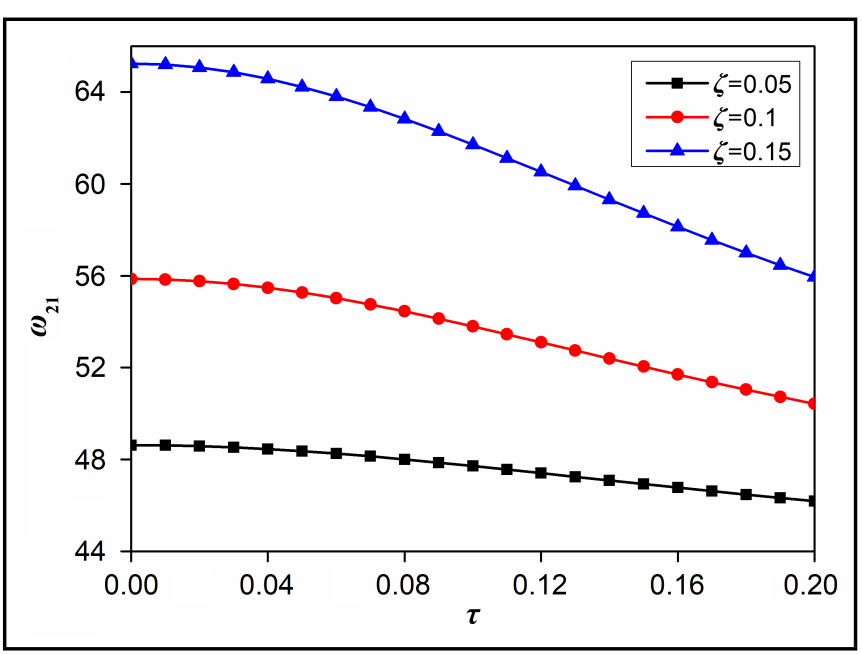

Figure 8. Variation of the second-order frequency of the functionally graded nanoplate with respect to the nonlocal parameter under different material characteristic scales $\left(\xi=1, \lambda=1, P_{x}=P_{y}=50\right)$.

rameter (i.e. increasingly steep change curve), which involves the coupling effect of the two scale parameters because the magnitude of material characteristic scales can influence the changing rate of the nonlocal effect.

In order to explore the effect of the material characteristic scale on natural frequencies, taking the nonlocal parameter as $0.05,0.1,0.15$, respectively, we obtain the relationship between the dimensionless natural frequencies and the material characteristic scale as illustrated in Figs. 9-10. When different nonlocal parameters are adopted, the natural frequency is continuously increasing as the material characteristic scale increases. So the stiffness of the nanoplate is constantly increasing, which reflects the stiffness hardening effect. Similar to Figs. 3-4, the phenomenon that the internal characteristic scale parameters have a greater influence on the higher-order frequencies than lower-order frequencies also appears in Figs. 910.

Similarly, we can reveal the effect of the dimensionless axial tensions $P_{x}$ and $P_{y}$ on dimensionless natural frequencies in Figs. 11-12, where the nonlocal parameter 0.1 is adopted. Therefore, we obtain variations of the first two frequencies of 


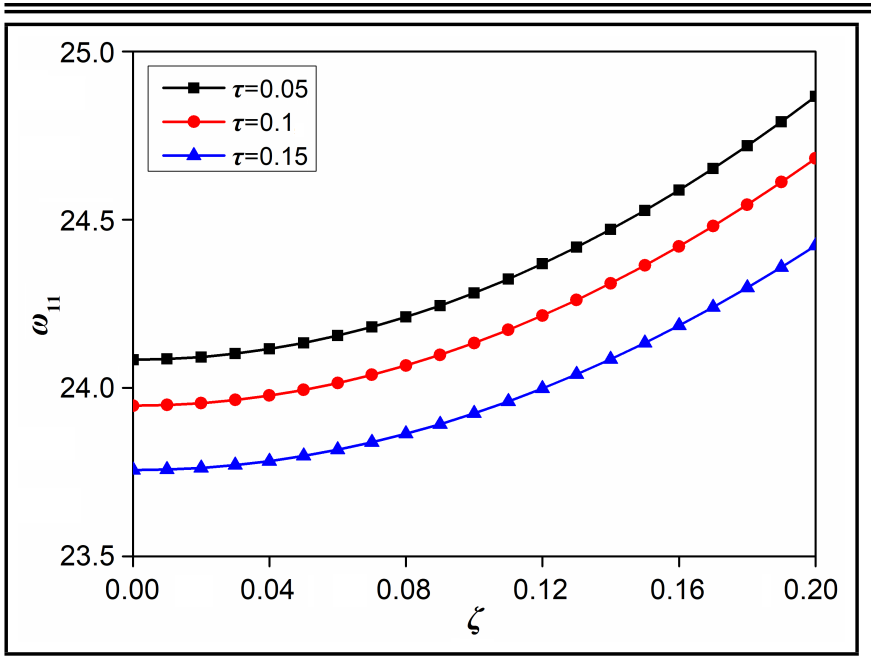

Figure 9. Variation of the first-order frequency of the functionally graded nanoplate with respect to the material characteristic scale under different nonlocal parameters $\left(\xi=1, \lambda=1, P_{x}=P_{y}=50\right)$.

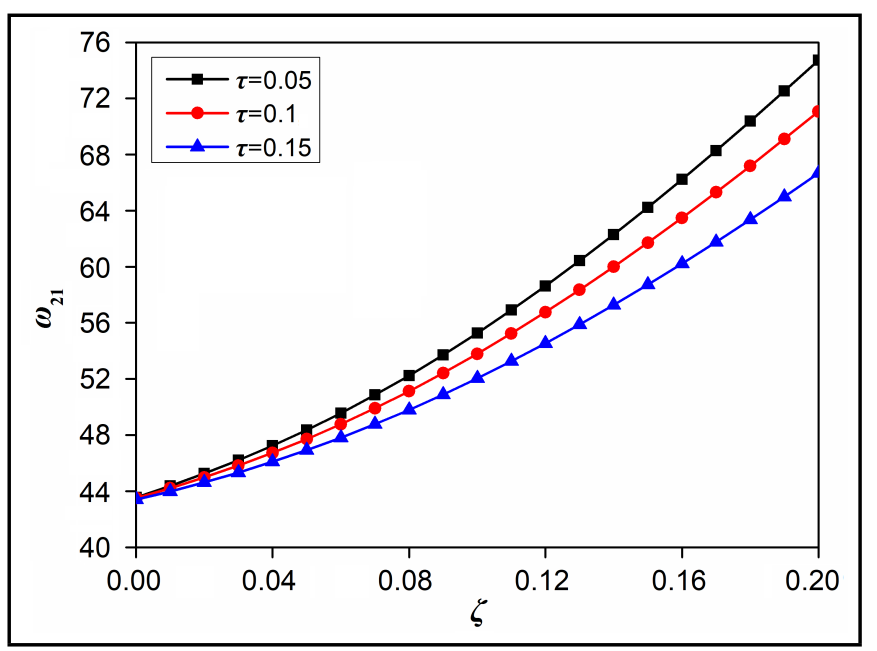

Figure 10. Variation of the second-order frequency of the functionally graded nanoplate with respect to the material characteristic scale under different nonlocal parameters $\left(\xi=1, \lambda=1, P_{x}=P_{y}=50\right)$.

the functionally graded nanoplate with respect to the material characteristic scale under different tensions. It is seen that the greater the tensions, the greater the natural frequencies, which reflects the initial tension play a role in enhancing the stiffness of the nanoplate while the material characteristic scale also has a stiffness hardening effect. Consequently, it can be considered to increase the tensions in $x$ and $y$ directions to achieve the effect of enhancing the stiffness of the functionally graded nanoplate. Note that the initial biaxial tension may be caused by increasing the ambient temperature, so a higher change of temperature can result in a larger vibration natural frequency of the axially moving functionally graded nanoplate.

Finally, to explore the coupling effect of the nonlocal parameter and the material characteristic scale, we take the material characteristic scale $\zeta$ equal to $0.05,0.08,0.1$, respectively. The results are exhibited in Fig. 13, from which we can analyze the influence of the ratio $\tau / \zeta$ on the natural frequency. It can be seen that when $\tau / \zeta$ is less than 1 , that is $\zeta>\tau$, the natural frequency increases with the increase of the material characteristic scale. At this stage, the material characteristic scale, namely the strain gradient effect plays a dominant role,

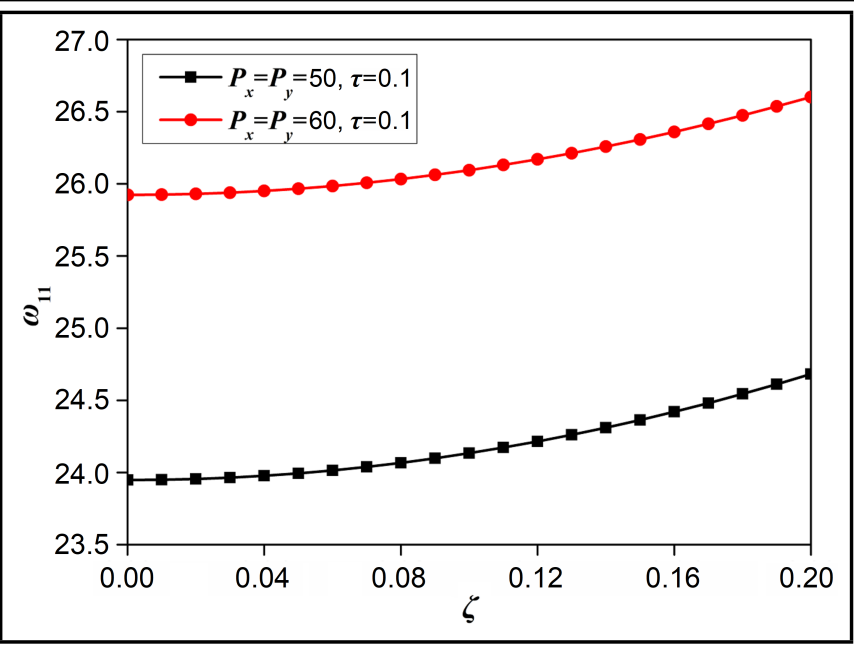

Figure 11. Variation of the first-order frequency of the functionally graded nanoplate with respect to the material characteristic scale under different tensions $(\xi=1, \lambda=1)$.

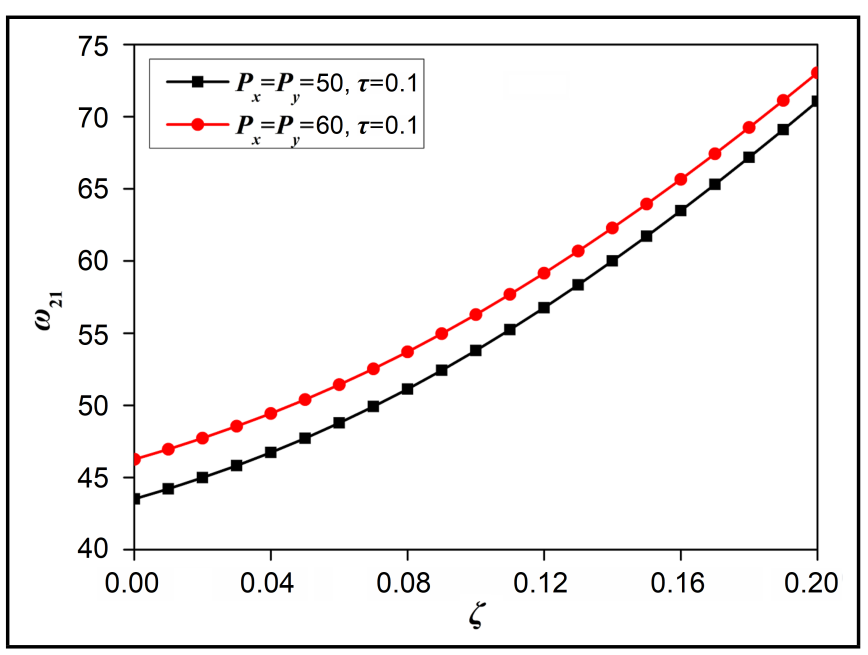

Figure 12. Variation of the second-order frequency of the functionally graded nanoplate with respect to the material characteristic scale under different tensions $(\xi=1, \lambda=1)$.

and the increase in the natural frequency corresponds to the stiffness hardening behaviour. When $\tau / \zeta$ is equal to 1 , that is, $\tau=\zeta$, the frequency degenerates to the natural frequency of the classic thin plate. When $\tau / \zeta$ is larger than 1 , that is $\tau>\zeta$, the natural frequency decreases with the increase of the material characteristic scale, where the nonlocal parameter namely the nonlocal effect manages the stiffness softening behaviour. As we know, stiffness softening and hardening phenomena were widely found in the nanostructural dynamics. In fact, the coupling effect of the same magnitude of the nonlocal parameter and the material characteristic scale results in the behaviour of stiffness softening and hardening behaviours. In the nonlocal strain gradient theory, the nonlocal parameter and the material characteristic scale are on the same order of magnitude, and the nonlocal effect and strain gradient effect respectively represented by the two are also equivalent. In addition, as mentioned above, there is a coupling effect between the two internal characteristic scale parameters, that is, the specific value of one parameter affects the effect of another parameter on the dynamic behaviour of nanostructures. It is the same magnitude coupling effect between the two internal 
characteristic scale parameters that leads to the formation of softening and stiffening mechanisms in the mechanical properties of functionally graded nanoplates. In fact, in addition to the softening and stiffening mechanism, there is a third special case where the stiffness keeps a constant. At this stage, the nonlocal parameter is equal to the material characteristic scale, thus the softening mechanism of nonlocal scale effect counteracts the stiffening mechanism of strain gradient effect, and the classical vibration results are recovered from the nonlocal strain gradient theory.

\section{CONCLUSIONS}

The axially moving functionally graded nanoplate model is developed through the nonlocal strain gradient approach. The initial biaxial tensile force is considered and the governing equation including the effects of nonlocal, strain gradient, axial motion, biaxial tension, aspect ratio and so on is derived. The numerical results of natural frequencies are determined using the complex modal method. The natural frequencies of axially moving functionally graded nanoplates become smaller with the increases of axial speed and the decreasing rate gets faster with a higher axial speed. Hence increasing axial speed of the nanoplate may cause the instability behaviour. The greater the aspect ratio is, the larger the frequency is. Hence natural frequencies of a square nanoplate are relatively low. The natural frequency increases with the increase of biaxial tensions. The natural frequencies decrease with the increase of the nonlocal parameter, while increase with the increase of the material characteristic scale. The nonlocal parameter and material characteristic scale are coupled in micro-dynamic mechanisms. When the nonlocal parameter/material characteristic scale is larger than the other, the nonlocal effect/strain gradient effect dominates the free vibration of axially moving functionally graded nanoplates, respectively showing the softening and stiffening behaviours of equivalent bending rigidity. When the nonlocal parameter is equal to the material characteristic scale, the equivalent bending rigidity remains unchanged and is equal to the corresponding classical result. Hence the study confirms that the nonlocal scale effect and the strain gradient effect are at the same level in internal characteristic effects included in the nonlocal strain gradient theory.

\section{ACKNOWLEDGEMENTS}

This work was supported by the Open Project of MOE Key Lab of Disaster Forecast and Control in Engineering (Jinan University, Grant No. 20180930002), and by the National Natural Science Foundation of China (Grant Nos. 51875374 and 11972240).

\section{REFERENCES}

1 Yan, J. W., Liew, K. M., and He, L. H. Free vibration analysis of single-walled carbon nanotubes using a higher-order gradient theory, Journal of Sound and Vibration, 332 (15), 3740-3755, (2013). https://dx.doi.org/10.1016/j.jsv.2013.02.004

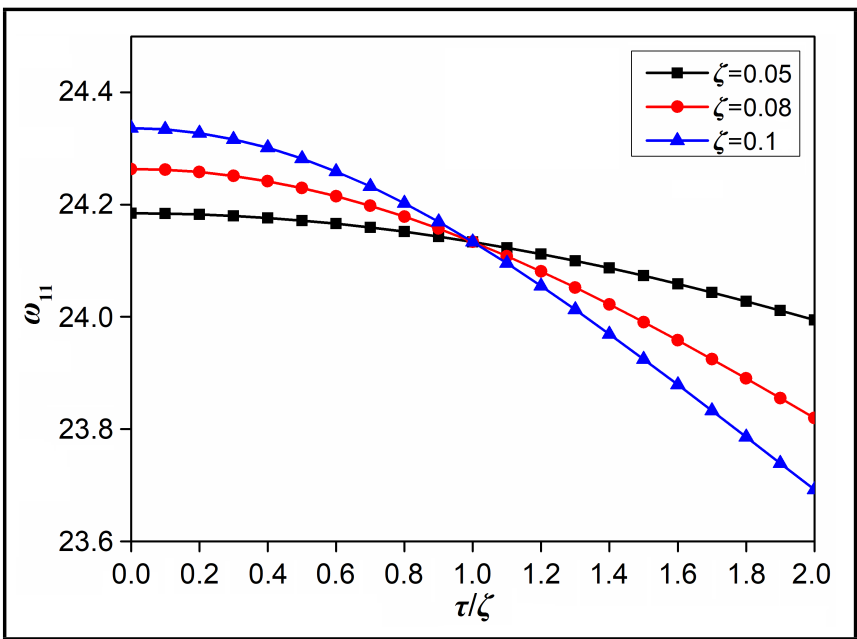

Figure 13. Variation of the first-order frequency of the functionally graded nanoplate with the ratio of the nonlocal parameter to material characteristic scale $\left(\xi=1, \lambda=1, P_{x}=P_{y}=50\right)$.

2 Bozyigit, D., Yazdani, N., Yarema, M., Yarema, O., Lin, W. M. M., Volk, S., Vuttivorakulchai, K., Luisier, M., Juranyi, F., and Wood, V. Soft surfaces of nanomaterials enable strong phonon interactions, Nature, 531 (7596), 618622, (2016). https://dx.doi.org/10.1038/nature16977

3 Iijima, S. Helical microtubules of graphitic carbon, Nature, 354, 56-58, (1991). https://dx.doi.org/10.1038/354056a0

4 Novoselov, K. S., Geim, A. K., Morozov, S. V., Jiang, D., Zhang, Y., Dubonos, S. V., Grigorieva, I. V., and Firsov, A. A. Electric field effect in atomically thin carbon films, Science, 306 (5696), 666-669, (2004). https://dx.doi.org/10.1126/science.1102896

5 Milledge, H. J., Nave, E., and Weller, F. H. Transformation of cubic boron nitride to a graphitic form of hexagonal boron nitride, Nature, 184 (4687), 715, (1959). https://dx.doi.org/10.1038/184715a0

6 Wong, E. W., Sheehan, P. E., and Lieber, C. M. Nanobeam mechanics: Elasticity, strength, and toughness of nanorods and nanotubes, Science, 277 (5334), 1971-1975, (1997). https://dx.doi.org/10.1126/science.277.5334.1971

7 Ansari, R., Sahmani, S., and Arash, B. Nonlocal plate model for free vibrations of single-layered graphene sheets, Physics Letters A, 375 (1), 53-62, (2010). https://dx.doi.org/10.1016/j.physleta.2010.10.028

8 Mirzaei, Y. Exact size-dependent elasticity solution for free radial vibration of nano-sphere, International Journal of Acoustics and Vibration, 24 (4), 687-692, (2019). https://dx.doi.org/10.20855/ijav.2019.24.41393

9 Liew, K. M., Pan, Z. Z., and Zhang, L. W. The recent progress of functionally graded CNT reinforced composites and structures, Science China Physics, Mechanics \& Astronomy, 63 (3), 234601, (2020). https://dx.doi.org/10.1007/s11433-019-1457-2 
10 Numanoglu, H. M. and Civalek, O. On the dynamics of small-sized structures, International Journal of Engineering Science, 145, 103164, (2019). https://dx.doi.org/10.1016/j.ijengsci.2019.103164

11 Thai, H. T., Vo, T. P., Nguyen, T. K., and Kim, S. E. A review of continuum mechanics models for size-dependent analysis of beams and plates, Composite Structures, 177, 196-219, (2017). https://dx.doi.org/10.1016/j.compstruct.2017.06.040

12 Ansari, R. and Sahmani, S. Prediction of biaxial buckling behavior of single-layered graphene sheets based on nonlocal plate models and molecular dynamics simulations, Applied Mathematical Modelling, 37 (12-13), 7338-7351, (2013). https://dx.doi.org/10.1016/j.apm.2013.03.004

13 Eringen, A. C. Part III-Nonlocal polar field theories, Continuum Physics, Academic Press, New York, 205-267, (1976). https://dx.doi.org/10.1016/B978-0-12240804-5.50009-9

14 Eringen, A. C. and Edelen, D. G. B. On nonlocal elasticity, International Journal of Engineering Science, 10, 233-248, (1972). https://dx.doi.org/10.1016/0020-7225(72)90039-0

15 Eringen, A. C. On differential equations of nonlocal elasticity and solutions of screw dislocation and surface waves, Journal of Applied Physics, 54 (9), 4703-4710, (1983). https://dx.doi.org/10.1063/1.332803

$16 \mathrm{Xu}, \mathrm{X}$. J. and Deng, Z. C. Surface effects of adsorptioninduced resonance analysis on micro/nanobeams via nonlocal elasticity, Applied Mathematics and Mechanics-English Edition, 34, 37-44, (2013). https://dx.doi.org/10.1007/s10483-013-1651-9

17 Lim, C. W., Islam, M. Z., and Zhang, G. A nonlocal finite element method for torsional statics and dynamics of circular nanostructures, International Journal of Mechanical Sciences, 94-95, 232-243, (2015). https://dx.doi.org/10.1016/j.ijmecsci.2015.03.002

18 Yang, W. D., Kang, W. B., and Wang, X. Thermal and surface effects on the pull-in characteristics of circular nanoplate NEMS actuator based on nonlocal elasticity theory, Applied Mathematical Modelling, 43, 321-336, (2017). https://dx.doi.org/10.1016/j.apm.2016.11.023

19 Jalaei, M. H. and Arani, A. G. Size-dependent static and dynamic responses of embedded doublelayered graphene sheets under longitudinal magnetic field with arbitrary boundary conditions, Composites Part B-Engineering, 142, 117-130, (2018). https://dx.doi.org/10.1016/j.compositesb.2017.12.053

20 Yang, Y. and Lim, C. W. Non-classical stiffness strengthening size effects for free vibration of a nonlocal nanostructure, International Journal of Mechanical Sciences, 54 (1), 57-68, (2012). https://dx.doi.org/10.1016/j.ijmecsci.2011.09.007
21 Li, C., Lim, C. W., and Yu, J. L. Dynamics and stability of transverse vibrations of nonlocal nanobeams with a variable axial load, Smart Materials \& Structures, 20 (1), 015023, (2011). https://dx.doi.org/10.1088/0964-1726/20/1/015023

22 Shen, J. P. and Li, C. A semi-continuum-based bending analysis for extreme-thin micro/nano-beams and new proposal for nonlocal differential constitution, Composite Structures, 172, 210-220, (2017). https://dx.doi.org/10.1016/j.compstruct.2017.03.070

23 Lim, C. W., Zhang, G., and Reddy, J. N. A higherorder nonlocal elasticity and strain gradient theory and its applications in wave propagation, Journal of the Mechanics and Physics of Solids, 78, 298-313, (2015). https://dx.doi.org/10.1016/j.jmps.2015.02.001

$24 \mathrm{Li}, \mathrm{L}$. and $\mathrm{Hu}, \mathrm{Y}$. J. Torsional vibration of bi-directional functionally graded nanotubes based on nonlocal elasticity theory, Composite Structures, 172, 242-250, (2017). https://dx.doi.org/10.1016/j.compstruct.2017.03.097

25 Sahmani, S. and Aghdam, M. M. Nonlinear instability of axially loaded functionally graded multilayer graphene platelet-reinforced nanoshells based on nonlocal strain gradient elasticity theory, International Journal of Mechanical Sciences, 131, 95-106, (2017). https://dx.doi.org/10.1016/j.ijmecsci.2017.06.052

26 Sahmani, S., Aghdam, M. M., and Rabczuk, T. Nonlocal strain gradient plate model for nonlinear large-amplitude vibrations of functionally graded porous micro/nano-plates reinforced with GPLs, Composite Structures, 198, 51-62, (2018). https://dx.doi.org/10.1016/j.compstruct.2018.05.031

27 Apuzzo, A., Barretta, R., Faghidian, S. A., Luciano, R., and de Sciarra, F. M. Free vibrations of elastic beams by modified nonlocal strain gradient theory, International Journal of Engineering Science, 133, 99-108, (2018). https://dx.doi.org/10.1016/j.ijengsci.2018.09.002

28 Sahmani, S. and Aghdam, M. M. Nonlinear primary resonance of micro/nano-beams made of nanoporous biomaterials incorporating nonlocality and strain gradient size dependency, Results in Physics, 8, 879-892, (2018). https://dx.doi.org/10.1016/j.rinp.2018.01.002

29 Chen, W., Wang, L., and Dai, H. L. Stability and nonlinear vibration analysis of an axially loaded nanobeam based on nonlocal strain gradient theory, International Journal of Applied Mechanics, 11 (7), 1950069, (2019). https://dx.doi.org/10.1142/S1758825119500698

30 Sahmani, S., Fattahi, A. M., and Ahmed, N. A. Analytical mathematical solution for vibrational response of postbuckled laminated FG-GPLRC nonlocal strain gradient micro/nanobeams, Engineering with Computers, 35 (4), 11731189, (2019). https://dx.doi.org/10.1007/s00366-018-06578 
31 Jalaei, M. H. and Civalek, O. A nonlocal strain gradient refined plate theory for dynamic instability of embedded graphene sheet including thermal effects, Composite Structures, 220, 209-220, (2019). https://dx.doi.org/10.1016/j.compstruct.2019.03.086

32 Sahmani, S., Fotouhi, M., and Aghdam, M. M. Sizedependent nonlinear secondary resonance of micro-/nanobeams made of nano-porous biomaterials including truncated cube cells, Acta Mechanica, 230 (3), 1077-1103, (2019). https://dx.doi.org/10.1007/s00707-018-2334-9

${ }^{33}$ Karami, B., Janghorban, M., and Rabczuk, T. Dynamics of two-dimensional functionally graded tapered Timoshenko nanobeam in thermal environment using nonlocal strain gradient theory, Composites Part B-Engineering, 182, 107622, (2020). https://dx.doi.org/10.1016/j.compositesb.2019.107622

${ }^{34}$ Mirjavadi, S. S., Afshari, B. M., Barati, M. R., and Hamouda, A. M. S. Transient response of porous inhomogeneous nanobeams due to various impulsive loads based on nonlocal strain gradient elasticity, International Journal of Mechanics and Materials in Design, 16 (1), 57-68, (2020). https://dx.doi.org/10.1007/s10999-019-09452-2

35 Mir, M. and Tahani, M. Graphene-based mass sensors: chaotic dynamics analysis using the nonlocal strain gradient model, Applied Mathematical Modelling, 81, 799-817, (2020). https://dx.doi.org/10.1016/j.apm.2020.01.022

${ }^{36}$ Malik, M. and Das, D. Free vibration analysis of rotating nano-beams for flap-wise, chord-wise and axial modes based on Eringen's nonlocal theory, International Journal of Mechanical Sciences, 179, 105655, (2020). https://dx.doi.org/10.1016/j.ijmecsci.2020.105655

37 Kiani, K. Longitudinal, transverse, and torsional vibrations and stabilities of axially moving single-walled carbon nanotubes, Current Applied Physics, 13 (8), 1651-1660, (2013). https://dx.doi.org/10.1016/j.cap.2013.05.008

38 Yao, L. Q., Ji, C. J., Shen, J. P., and Li, C. Free vibration and wave propagation of axially moving functionally graded Timoshenko microbeams, Journal of the Brazilian Society of Mechanical Sciences and Engineering, 42, 137, (2020). https://dx.doi.org/10.1007/s40430-020-2206-9

39 Pham, P. T. and Hong, K. S. Dynamic models of axially moving systems: A review, Nonlinear Dynamics, 100 (1), 315-349, (2020). https://dx.doi.org/10.1007/s11071-02005491-z

40 Koizumi, M. FGM activities in Japan, Composites Part B-Engineering, 28 (1-2), 1-4, (1997). https://dx.doi.org/10.1016/S1359-8368(96)00016-9
41 Qatu, M. S. Free vibration of laminated composite rectangular plates, International Journal of Solids and Structures, 28 (8), 941-954, (1991). https://dx.doi.org/10.1016/00207683(91)90122-V

42 Uymaz, B., Aydogdu, M., and Filiz, S. Vibration analyses of FGM plates with in-plane material inhomogeneity by Ritz method, Composite Structures, 94 (4), 1398-1405, (2012). https://dx.doi.org/10.1016/j.compstruct.2011.11.002

43 Basmaci, A. N., Filiz, S., and Sahin, M. Experimental analysis of welded rods with a functionally graded material approach, Applied Sciences-Basel, 10 (11), 3908, (2020). https://dx.doi.org/10.3390/app10113908

44 Aksencer, T. and Aydogdu, M. Levy type solution method for vibration and buckling of nanoplates using nonlocal elasticity theory, Physica E, 43 (4), 954-959, (2011). https://dx.doi.org/10.1016/j.physe.2010.11.024

45 Sahmani, S., Aghdam, M. M., and Rabczuk, T. A unified nonlocal strain gradient plate model for nonlinear axial instability of functionally graded porous micro/nano-plates reinforced with graphene platelets, Materials Research Express, 5 (4), 045048, (2018). https://dx.doi.org/10.1088/2053-1591/aabdbb

46 Shen, J. P., Wang, P. Y., Gan, W. T., and Li, C. Stability of vibrating functionally graded nanoplates with axial motion based on the nonlocal strain gradient theory, International Journal of Structural Stability and Dynamics, 20 (8), 2050088, (2020). https://dx.doi.org/10.1142/S0219455420500881

47 Lu, L., Yang, X. D., Zhang, W., and Lai, S. K. On travelling wave modes of axially moving string and beam, Shock and Vibration, 2019, 9496180, (2019). https://dx.doi.org/10.1155/2019/9496180

48 Liu, J. J., Li, C., Fan, X. L., and Tong, L. H. Transverse free vibration and stability of axially moving nanoplates based on nonlocal elasticity theory, Applied Mathematical Modelling, 45, 65-84, (2017). https://dx.doi.org/10.1016/j.apm.2016.12.006

49 Jalaei, M. H. and Thai, H. T. Dynamic stability of viscoelastic porous FG nanoplate under longitudinal magnetic field via a nonlocal strain gradient quasi-3D theory, Composites Part B-Engineering, 175, 107164, (2019). https://dx.doi.org/10.1016/j.compositesb.2019.107164

50 Aydogdu, M., Arda, M., and Filiz, S. Vibration of axially functionally graded nano rods and beams with a variable nonlocal parameter, $A d$ vances in Nano Research, 6 (3), 257-278, (2018). https://dx.doi.org/10.12989/anr.2018.6.3.257 